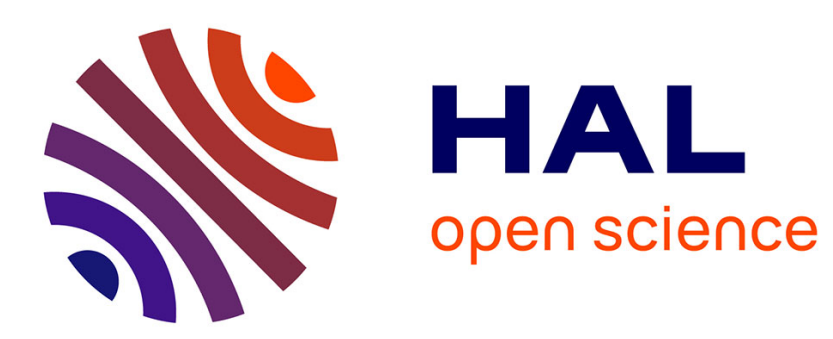

\title{
Modeling and Decentralized Control for the Multiple UAVs Formation based on Lyapunov design and redesign
}

\author{
Zhicheng Hou, Isabelle Fantoni, Arturo Zavala-Río
}

\section{To cite this version:}

Zhicheng Hou, Isabelle Fantoni, Arturo Zavala-Río. Modeling and Decentralized Control for the Multiple UAVs Formation based on Lyapunov design and redesign. 2nd IFAC Workshop on Research, Education and Development of Unmanned Aerial Systems (RED UAS 2013), Nov 2013, Compiègne, France. pp.337-344. hal-00932861

\section{HAL Id: hal-00932861 https://hal.science/hal-00932861}

Submitted on 17 Jan 2014

HAL is a multi-disciplinary open access archive for the deposit and dissemination of scientific research documents, whether they are published or not. The documents may come from teaching and research institutions in France or abroad, or from public or private research centers.
L'archive ouverte pluridisciplinaire HAL, est destinée au dépôt et à la diffusion de documents scientifiques de niveau recherche, publiés ou non, émanant des établissements d'enseignement et de recherche français ou étrangers, des laboratoires publics ou privés. 


\title{
Modeling and Decentralized Control for the Multiple UAVs Formation based on Lyapunov design and redesign
}

\author{
Zhicheng HOU * Isabelle FANTONI* Arturo ZAVALA-RIO ** \\ * Université de Technologie de Compiègne - CNRS, UMR 7253 \\ Heudiasyc,60200 Compiègne, France (e-mail: zhicheng.hou@hds.utc.fr, \\ isabelle.fantoni@hds.utc.fr, corresponding author) \\ ** IPICYT, Camino a la Presa San José 2055, Lomas 4a. Sección \\ 78216, San Luis Potosí, Mexico (e-mail: azavala@ipicyt.edu.mx)
}

\begin{abstract}
This paper concerns the leader-follower multiple agent formation with nonlinear and coupled individual dynamics. We address the problem of multi-agent formation control by proposing a decentralized control strategy. The agents in the formation are quad-rotors UAVs. By attributing the high-order nonlinear and unmodelled dynamics as uncertainties, we propose a switching singular system model to represent the formation of the multiple UAVs system with switching topology. For stabilizing the system with uncertainties, the decentralized controllers are designed by using Lyapunov design and redesign. The simulation results illustrate the stated problems and verify the proposed theorem.
\end{abstract}

Keywords: Multi-agent systems, Leader-follower, Modeling, Decentralized control, Uncertainty, Lyapunov design and redesign

\section{INTRODUCTION}

The research on Multi-Agent Systems (MAS) has attracted an increasing attention over the last few years because of the applications on formation control, distributed sensor network, flocking, distributed computation and synchronization of coupled chaotic oscillators, see You et al. (2013). In particular, numerous extensions to the consensus protocol for multi-robots have been proposed, such as the works of Porfiri et al. (2007), Ni and Cheng (2010), and Li et al. (2013). These researches are motivated by the widespread applications, such as cooperative searching, scheduling of automated highway systems, air traffic control, terrain and utilities inspection, disaster monitoring, environmental surveillance, rescue and planetary exploration.

In the past few years, there have been numerous results concerning the cooperative control of the simple linear (first-order or second-order) multi-agent systems, such as Porfiri et al. (2007), Vela et al. (2008), Qu et al. (2008), Ni and Cheng (2010), Wang and $\mathrm{Hu}$ (2010), Guo et al. (2011), and Cao et al. (2012). Most of the researchers propose their ideas with the assumption that the model of agent is linear or simplified. In practice, the dynamics of the agents are usually nonlinear. Under this circumstance, the agents may not achieve consensus in the presence of disturbance sometimes.

\footnotetext{
^ This work was carried out in the framework of the Labex MS2T, which was funded by the French Government, through the program "Investments for the future" managed by the National Agency for Research (Reference ANR-11-IDEX-0004-02)
}

The consensus problem has been studied in many papers. A different dynamics between the leader and the followers has been considered by Hong et al. (2006), where the tracking problem without detecting the velocity of the leader has been solved. Ni and Cheng (2010) used the Riccati and Lyapunov inequalities to design the controller for a group with switched topology. They have found that the multiagents system is stable if the graph is jointly connected. Notarstefano et al. (2011) have studied the group with a stationary leader in the case of communication connecting intermittently. Cao et al. (2012) focus on the group with a directed graph, which is strong connected. They also discussed the observability of the tracking errors between the leader and the followers.

In this paper, the problem of consensus is also discussed, but the distinction is that the model of each agent in the group is nonlinear and coupled. The unmodeled dynamics are considered in the controller design. We introduce our leader-follower formation with quad-rotors UAVs agents, where the switching topology is taken into account. There are several cases that cause the switching of the topology, for instance, the changing of the neighbors, agents entering and leaving the group, and the changing of the leaders. Considering the property of the agents, our objective is to develop a decentralized formation strategy by designing the controller based on Lyapunov design and redesign, which is mainly inspired by Memon and Khalil (2008). In this work, the neighbors information is only needed instead of the global information. The model of the formation is represented by a switching system, which facilitates the stability proof. Sometimes, the switching system may be singular. Even we focus on the scenario of leader-follower formation in the present work, which is traditionally 
considered as a centralized formation, we devote to develop a decentralized strategy. In this paper, the leaders are variable and they comply with not only the reference signal, but also the followers. Additionally, the number of leaders is not restricted to one. These constitute our main contribution.

\section{PRELIMINARIES}

\subsection{Graph theory notions and some notations}

A leader-follower formation group can be represented by the directed graph $\mathcal{G}=(\mathcal{V}, \mathcal{E})$. The vertex set $\mathcal{V}=$ $\{1,2, \ldots, n\}$ contains the indexes of the agents whose collection is in future represented by $\mathbf{S}=\left\{s_{1}, s_{2}, \ldots, s_{n}\right\}$. The set $\mathbf{S}$ represents the collection of the agents. Let us denote by $\mathbf{S}_{L} \subset \mathbf{S}$, which contains all the leaders. The set of followers is represented by $\mathbf{S}_{F}$ satisfying $\mathbf{S}_{l} \cap \mathbf{S}_{F}=\mathbf{S}$. $\mathcal{E} \subseteq \mathcal{V} \times \mathcal{V}$ is the edge set. The neighbors of agent $s_{i}$ are denoted by $\mathcal{N}_{i}=\{j \in \mathcal{V}:(i, j) \in \mathcal{E}\}$.

\subsection{Switching topology}

Let us assume that there are totally $m$ possible graphs $\sum_{\mathcal{G}}=\left\{\mathcal{G}_{1}, \mathcal{G}_{2}, \ldots, \mathcal{G}_{m}\right\}$, which represent the communicating topologies of the agents. The corresponding set of switching time instants is $\sigma_{t}=\left\{t_{1}, \ldots, t_{k}\right\}$. The formation of the agents can be specified by one of these graphs at each time instant. A piecewise right continuous function is defined as follows

$$
\sigma(t):[0, \infty) \rightarrow \Psi, \text { where } \Psi=\{1, \ldots, m\}
$$

We call this function a switching signal which indicates the graph currently activated. The set $\Psi$ aggregates the indexes of all the possible graphs.

Definition 1. The consensus for a MAS with leaderfollower formation strategy is achieved, if the following condition is satisfied $\left\|\left[\mathcal{X}_{1}-r(t), \ldots, \mathcal{X}_{n}-r(t)\right]^{T}\right\| \rightarrow 0$, where $\mathcal{X}_{i}$ is the states vector of agent $s_{i}, i=\{1, \ldots, n\}$. The notation $\|\cdot\|$ is the 2 -norm in $\mathbb{R}^{2}$.

\section{MODELING OF MULTIPLE UAVS SYSTEM}

\subsection{Uncertainty analysis for a single quad-rotor $U A V$}

In most of the articles, for example, Vela et al. (2008), Wang and $\mathrm{Hu}$ (2010), Guo et al. (2011), and Cao et al. (2012), simplified agent models is used for the convenience of controller design and stability analysis. However, in some cases, the nonlinear influence is not negligible. Especially for the model of quad-rotor UAVs, which is nonlinear and coupled, sometimes the assumption conditions for reducing the model will not be satisfied in presence of disturbance or unmodeled dynamics. In this case, the consensus algorithm always fails to work (we will illustrate it in the section of simulation). To deal with these disadvantages, we present in this section the accurate model for each UAV and then, both the simplified model and the unmodeled dynamics, which is caused by the model reduction, will be used in the controller design and stability analysis.
The dynamics of the quad-rotors UAV is given as Bresciani (2008)

$$
\begin{aligned}
\ddot{X} & =(\sin \psi \sin \phi+\cos \psi \sin \theta \cos \phi) \frac{F_{T}}{m_{F}} \\
\ddot{Y} & =(-\cos \psi \sin \phi+\sin \psi \sin \theta \cos \phi) \frac{F_{T}}{m} \\
\ddot{Z} & =-g+(\cos \theta \cos \phi) \frac{F_{T}}{m} \\
\dot{p} & =\frac{I_{Y Y}-I_{Z Z}}{I_{X X}} q r-\frac{J_{T P}}{I_{X X}} q \Omega+\frac{\tau_{\phi}}{I_{X X}} \\
\dot{q} & =\frac{I_{Z Z}-I_{X X}}{I_{Y Y}} p r+\frac{J_{T P}}{I_{Y Y}} p \Omega+\frac{\tau_{\theta}}{I_{Y Y}} \\
\dot{r} & =\frac{I_{X X}-I_{Y Y}}{I_{Z Z}} p q+\frac{\tau_{\psi}}{I_{Z Z}}
\end{aligned}
$$

where $(X, Y, Z)$ are the coordinates of the UAV in the inertial frame. $\phi, \theta$ and $\psi$ represent the Euler angles. $p, q, r$ are the angular velocities under the body-fixed frame, and $\Omega=\omega_{2}+\omega_{4}-\omega_{1}-\omega_{3}$ is the overall propellers speed, where $\omega_{i}(i=1,2,3,4)$ is the speed of each rotor. $F_{T}$ is the total thrust, $\tau_{\theta}$ is the pitching torque, $\tau_{\phi}$ is the rolling torque and $\tau_{\psi}$ is the yawing torque. $I_{X X}, I_{Y Y}$, and $I_{Z Z}$ are moments of inertia corresponding to the axis of the body-fixed frame. $J_{T P}$ is the rotor inertia. The attitude rotating speed relation between the body coordinates and the generalized coordinates can be written as follows

$$
\left[\begin{array}{l}
\dot{\phi} \\
\dot{\theta} \\
\dot{\psi}
\end{array}\right]=\left[\begin{array}{ccc}
1 & \sin \phi \tan \theta & \cos \phi \tan \theta \\
0 & \cos \phi & -\sin \phi \\
0 & \sin \phi / \cos \theta & \cos \phi / \cos \theta
\end{array}\right]\left[\begin{array}{l}
p \\
q \\
r
\end{array}\right]
$$

Assumption 1. The altitude of each agent is slow-varying during the formation control period.

Assumption 2. The attitude angle is less than $\pi / 2$ and the input forces or torques (thrust, pitch, roll and yaw) are bounded.

Assumption 3. The attitude angles will be closed to zero.

Assumption 1 guarantees that the pitch angle $|\theta|<\frac{\pi}{2}$ to avoid the elements become infinity. From assumption 2, the rotating matrix in (2) is invertible.

Let us denote

$$
o(\phi, \theta)=\left[\begin{array}{ccc}
0 & \sin \phi \tan \theta & \cos \phi \tan \theta \\
0 & \cos \phi-1 & -\sin \phi \\
0 & \sin \phi / \cos \theta & \cos \phi / \cos \theta-1
\end{array}\right]
$$

Using (3), the time derivative of (2) yields

$$
\left[\begin{array}{c}
\ddot{\phi} \\
\ddot{\theta} \\
\ddot{\psi}
\end{array}\right]=\left[\begin{array}{c}
\dot{p}+q r \\
\dot{q}-p r \\
\dot{r}+p q
\end{array}\right]+\bar{\Delta}_{R}
$$

where $\bar{\Delta}_{R}$ has the expression as follows

$$
\bar{\Delta}_{R}=o(\phi, \theta) \cdot\left[\begin{array}{c}
\dot{p} \\
\dot{q} \\
\dot{r}
\end{array}\right]+\dot{o}(\phi, \theta, \dot{\phi}, \dot{\theta}) \cdot\left[\begin{array}{l}
p \\
q \\
r
\end{array}\right]-\left[\begin{array}{l}
q r \\
-p r \\
p q
\end{array}\right]
$$

Therefore, the rotating dynamic using (1) and (2) is given by 


$$
\begin{aligned}
{\left[\begin{array}{c}
\ddot{\phi} \\
\ddot{\theta} \\
\ddot{\psi}
\end{array}\right]=} & {\left[\begin{array}{c}
\frac{\tau_{\phi}}{I_{\gamma_{\theta}}} \\
\frac{I_{\gamma_{\psi}}}{I_{Z Z}}
\end{array}\right]+\left[\begin{array}{l}
\frac{I_{X X}+I_{Y Y}-I_{Z Z}}{I_{X X}} q r-\frac{J_{T P}}{I_{X X}} q \Omega \\
\frac{I_{Z Z}-I_{X X}-I_{Y Y}}{I_{Y Y}} p r+\frac{J_{T P}}{I_{Y Y}} p \Omega \\
\frac{I_{X X}-I_{Y Y}+I_{Z Z}}{I_{Z Z}} p q
\end{array}\right] } \\
& +\bar{\Delta}_{R}
\end{aligned}
$$

According to assumption 1, we assume that the altitude is stabilized and the thrust force $F_{T}$ is approximately a constant. Therefore, the UAV works in a planar surface $X O Y$. Let us note that the uncertainty caused by assumption 1 is $\Delta_{F_{T}}$. The nominal value of thrust force is $\bar{F}_{T}$. By considering the definition of Euler angles, we can calculate the uncertainty $F_{T}=\frac{m g}{\cos (\theta) \cos (\phi)}=\bar{F}_{T}+\Delta_{F_{T}}$, where $\bar{F}_{T}=m g$.

The uncertainties in translation yields

$$
\begin{aligned}
& \Delta_{T}=\left[\begin{array}{l}
-\theta+\sin \psi \sin \phi+\cos \psi \sin \theta \cos \phi \\
\phi-\cos \psi \sin \phi+\sin \psi \sin \theta \cos \phi \\
-1+\cos \theta \cos \phi
\end{array}\right] \frac{F_{T}}{m} \\
& +\left[\begin{array}{l}
\frac{\Delta_{F_{T}}}{m_{F_{T}}} \cdot \theta \\
-\frac{\Delta_{F_{T}}}{m} \cdot \phi \\
\frac{\Delta_{F_{T}}}{m}
\end{array}\right]=\left[\begin{array}{l}
\Delta_{X} \\
\Delta_{Y} \\
\Delta_{Z}
\end{array}\right]
\end{aligned}
$$

By denoting $\bar{\Delta}_{R}=\left[\bar{\Delta}_{\phi}, \bar{\Delta}_{\theta}, \bar{\Delta}_{\psi}\right]^{T}$, the planar translational dynamic can be represented as

$$
\begin{aligned}
& \ddot{X}=\frac{\bar{F}_{T}}{m} \cdot \theta+\Delta_{X} \\
& \ddot{Y}=-\frac{\bar{F}_{T}}{m} \cdot \phi+\Delta_{Y} \\
& \ddot{\phi}=\frac{\tau_{\phi}}{I_{X X}}+\left(\frac{I_{X X}+I_{Y Y}-I_{Z Z}}{I_{X X}} q r-\frac{J_{T P}}{I_{X X}} q \Omega\right)+\bar{\Delta}_{\phi} \\
& \ddot{\theta}=\frac{\tau_{\theta}}{I_{Y Y}}+\left(\frac{I_{Z Z}-I_{X X}-I_{Y Y}}{I_{Y Y}} p r+\frac{J_{T P}}{I_{Y Y}} p \Omega\right)+\bar{\Delta}_{\theta} \\
& \ddot{\psi}=\frac{I_{X X}-I_{Y Y}+I_{Z Z}}{I_{Z Z}} p q+\frac{\tau_{\psi}}{I_{Z Z}}+\bar{\Delta}_{\psi}
\end{aligned}
$$

Since the planar dynamics in (7) have no zero dynamics from input $\tau_{\phi}$, respectively, $\tau_{\theta}$ to output, $Y$ respectively, $X$. It is possible to cancel the nonlinear dynamics by using feedback linearization. By setting the state vector $\mathcal{X}=[X, Y, \dot{X}, \dot{Y}, \phi, \theta, \psi, \dot{\phi}, \dot{\theta}, \dot{\psi}]^{T}$, the feedback linearization controller can be designed as,

$$
\begin{aligned}
& \tau_{\phi}=\alpha_{\phi}(\mathcal{X})+\beta_{\phi}(\mathcal{X}) u_{Y} \\
& \tau_{\theta}=\alpha_{\theta}(\mathcal{X})+\beta_{\theta}(\mathcal{X}) u_{X} \\
& \tau_{\psi}=\alpha_{\psi}(\mathcal{X})+\beta_{\psi}(\mathcal{X}) u_{\psi}
\end{aligned}
$$

where $\beta_{\phi}(\mathcal{X})=-\frac{I_{X X} m}{\bar{F}_{T}}, \beta_{\theta}(\mathcal{X})=\frac{I_{Y Y} m}{\bar{F}_{T}}$ and $\beta_{\psi}(\mathcal{X})=$ $I_{Z Z}$,

$$
\begin{aligned}
& \alpha_{\phi}(\mathcal{X})=-\left(I_{X X}+I_{Y Y}-I_{Z Z}\right) \dot{\theta} \dot{\psi}+J_{T P} q \Omega \\
& \alpha_{\theta}(\mathcal{X})=-\left(I_{Z Z}-I_{X X}-I_{Y Y}\right) \dot{\phi} \dot{\psi}-J_{T P} q \Omega \\
& \alpha_{\psi}(\mathcal{X})=-\left(I_{Z Z}+I_{X X}-I_{Y Y}\right) \dot{\phi} \dot{\theta}
\end{aligned}
$$

In the foregoing feedback linearization, the term $J_{T P} q \Omega$ is usually omitted in the practice, because the it is very small. Then, the planar dynamics in (7) can be rewritten as

$$
\begin{aligned}
& \ddot{X}=g \cdot \theta+\Delta_{X} \\
& \ddot{Y}=-g \cdot \phi+\Delta_{Y} \\
& \ddot{\phi}=-1 / g \cdot u_{Y}+\Delta_{\phi} \\
& \ddot{\theta}=1 / g \cdot u_{X}+\Delta_{\theta} \\
& \ddot{\psi}=u_{\psi}+\Delta_{\psi}
\end{aligned}
$$

where the uncertain parts $\Delta_{\phi}, \Delta_{\theta}$, and $\Delta_{\psi}$ are

$$
\begin{aligned}
\Delta_{\phi}= & \left(\frac{\cos \theta-\cos (2 \phi)}{\cos \theta} q r-q^{2} \frac{\sin \phi \cos \phi}{\cos \theta}+r^{2} \frac{\sin \phi \cos \phi}{\cos \theta}\right) \\
& \cdot\left(\frac{I_{X X}+I_{Y Y}-I_{Z Z}}{I_{X X}}\right)+\bar{\Delta}_{\phi} \\
\Delta_{\theta}= & {\left[\left(1-\frac{\cos \phi}{\cos \theta}\right) p r-2 q r \frac{\sin \phi \cos \phi \tan \theta}{\cos \theta}-r^{2} \frac{\cos ^{2} \phi \tan \theta}{\cos }\right.} \\
& \left.-p q \frac{\sin \phi}{\cos \theta}-q^{2} \sin \phi \tan \theta\right] \cdot\left(\frac{I_{Z Z}-I_{X X}-I_{Y Y}}{I_{Y Y}}\right) \\
& +\bar{\Delta}_{\theta}
\end{aligned}
$$

and

$$
\begin{aligned}
\Delta_{\psi}= & {\left[p r \sin \phi+r^{2} \sin \phi \cos \phi \tan \theta-q^{2} \sin \phi \cos \phi \tan \theta\right.} \\
& +p q(1-\cos \phi)+q r \cos (2 \phi) \tan \theta] \\
& \cdot\left(I_{X X}-I_{Y Y}+I_{Z Z}\right) / I_{Z Z}+\bar{\Delta}_{\psi}
\end{aligned}
$$

When the attitude angles (roll, pitch) keep varying in small range around the origin, the model uncertainties $\left(\Delta_{X}, \Delta_{Y}\right.$, $\Delta_{\phi}, \Delta_{\theta}$, and $\left.\Delta_{\psi}\right)$ are negligible. Thus, the dynamics of the UAV in the planar surface is decoupled into three subdynamics such that the dynamics in $X, Y$ directions and the yaw dynamics, which is shown as follows.

(1) Dynamics in $X$ direction

$$
\begin{aligned}
& \ddot{X}=g \cdot \theta+\Delta_{X} \\
& \ddot{\theta}=1 / g \cdot u_{X}+\Delta_{\theta}
\end{aligned}
$$

(2) Dynamics in $Y$ direction

$$
\begin{aligned}
& \ddot{Y}=-g \cdot \phi+\Delta_{Y} \\
& \ddot{\phi}=-1 / g \cdot u_{Y}+\Delta_{\phi}
\end{aligned}
$$

(3) Yaw dynamics

$$
\ddot{\psi}=u_{\psi}+\Delta_{\psi}
$$

In the present work, we always consider that the quadrotor UAVs fly with the same altitude and the yaw angles are always stabilized at zero. As the $X$ and $Y$ dynamics have the same structures, we just take the $X$ direction to develop our analysis.

Let us denote $x=\left[X, \dot{X}, \ddot{X}, X^{(3)}\right]^{T}$, then the state space expression will be

$$
\dot{x}=A x+B \delta_{i}
$$

where $\delta_{i}=u_{X}+\bar{F}_{T} / m \Delta_{\theta}+\ddot{\Delta}_{X}$, matrices $A$ and $B$ are as follows

$$
A=\left[\begin{array}{llll}
0 & 1 & 0 & 0 \\
0 & 0 & 1 & 0 \\
0 & 0 & 0 & 1 \\
0 & 0 & 0 & 0
\end{array}\right], B=\left[\begin{array}{l}
0 \\
0 \\
0 \\
1
\end{array}\right]
$$

Then, by using the feedback linearization controller (8), the nonlinear and coupled UAV model is decoupled as some subsystems, equation (11) is one of them. 
In summary of the above analysis, we can see that

(1) The UAV system has no nontrivial zero dynamics, the dynamics of the UAV can be reduced to a linear system by introducing feedback linearization.

(2) The dynamics along $x$ and $y$ axis are decoupled owing to feedback linearization.

(3) The pair $(A, B)$ is in controllable canonical form.

\subsection{Modeling of multiple UAVs system}

Let us denote $x_{i}$ as the state vector of the sub-dynamics of follower $s_{i}$. Note that in the following part, we say "the state of agent $s_{i}$ " instead of "the state vector of the subdynamics of follower $s_{i}$ in $X$ direction" for short. All of the relative variables are associated with the $X$ direction.If we use $u_{i}$ to replace $u_{X}$ in the following part, the equation (11) can be rewritten as

$$
\dot{x}_{i}=A x_{i}+B\left(u_{i}+\delta_{i}\right)
$$

Without considering the uncertainties, we get the nominal model of agent $s_{i}$ from (12).

$$
\dot{x}_{i}=A x_{i}+B u_{i}
$$

We define two kinds of errors in our work, they are

(a) Tracking errors between agents and the reference trajectory.

$$
d_{i}=x_{i}-\mathcal{R}(t)
$$

where $\mathcal{R}(t)^{T}=\left[r_{X}(t), \dot{r}_{X}(t), \ddot{r}_{X}(t), r_{X}^{(3)}(t)\right]$ is the reference signal, $d_{i}$ is the error between the state vector of agent $s_{i}$ and the reference $\mathcal{R}(t)$. Let us denote $\mathfrak{d}=\left[d_{1}^{T}, d_{2}^{T}, \ldots, d_{n}^{T}\right]^{T}$, where $\mathfrak{d} \in \mathbb{R}^{4 n}, n$ is the number of the agents in the group.

(b) Formation errors between agents and their neighbors.

$$
e_{i}=x_{i}-x_{i}^{n b}
$$

where $e_{i}$ is the error between the state vectors of agent $i$ and those of its neighbors. $x_{i}^{n b}$ satisfies

$$
x_{i}^{n b}=\sum_{j \in \mathcal{N}_{i}} \omega_{i j} x_{j}+\mu\left(s_{i}\right) \mathcal{R}(t)
$$

where $\omega_{i j}$ is a weight. $\mu\left(s_{j}\right)$ is given as follows

$$
\mu\left(s_{j}\right)= \begin{cases}0, & s_{i} \in S_{F} \\ \epsilon_{i}, & s_{i} \in S_{L}\end{cases}
$$

where $\epsilon_{i} \in(0,1]$. Let us denote $\mathfrak{e}=\left[e_{1}^{T}, e_{2}^{T}, \ldots, e_{n}^{T}\right]^{T}$ where $\mathfrak{e} \in \mathbb{R}^{4 n}$.

Remark 1. According to definition 1 , if the error $\mathfrak{d}$ defined in (a) converges to zero, the agents achieve consensus in the $X$ direction. Nevertheless, we can not directly design controllers to stabilize $\mathfrak{d}$, because not all of the agents are able to acquire the information of the reference. Only leaders can acquire the information of the reference. We can only design controllers to stabilize $\mathfrak{e}$.

In this paper, we use a mapping matrix to represent the relationship between $\mathfrak{d}$ and $\mathfrak{e}$. If we construct a mapping matrix $\mathcal{T}$ as

$$
\mathcal{T}(i j)=\left\{\begin{array}{cc}
-\omega_{i j} & i \neq j \\
1 & i=j
\end{array}\right.
$$

where $\omega_{i j} \in[0,1]$. For $i \in \mathcal{V}$ and $\left|\mathcal{N}_{i}\right| \neq 0$, the elements $\omega_{i j}(i, j \in \mathcal{V})$ satisfy

$$
\sum_{j=1}^{n} \omega_{i j}=\left\{\begin{array}{cc}
1 & s_{i} \in \mathbf{S}_{F} \\
1-\varepsilon_{i} & s_{i} \in \mathbf{S}_{L}
\end{array}\right.
$$

where $\varepsilon_{i} \in(0,1]$. Then, we have the following equation

$$
\left[\begin{array}{c}
x_{1}-\sum_{j \in \mathcal{N}_{1}} \omega_{1 j} x_{j} \\
\vdots \\
x_{n}-\sum_{j \in \mathcal{N}_{n}} \omega_{n j} x_{j}
\end{array}\right]-\left[\begin{array}{c}
\mu\left(s_{j}\right) \mathcal{R}(t) \\
\vdots \\
\mu\left(s_{j}\right) \mathcal{R}(t)
\end{array}\right]=\mathcal{T} \otimes I_{4}\left[\begin{array}{c}
x_{1}-\mathcal{R}(t) \\
\vdots \\
x_{n}-\mathcal{R}(t)
\end{array}\right]
$$

where $\otimes$ represents the Kronecker product.

If we note $\alpha(\mathcal{T})=\mathcal{T} \otimes I_{4}$, the mapping relating these two kinds of errors yields

$$
\mathfrak{e}=\alpha(\mathcal{T}) \mathfrak{d}
$$

where $\alpha(\mathcal{T}) \in \mathbb{R}^{4 n \times 4 n}$.

From (9), by neglecting $\delta_{i}$, the formation error dynamics for agent $s_{i}$ is given as follows

$$
\dot{e}_{i}=A e_{i}+B\left(u_{i}-\sum_{j \in \mathcal{N}_{i}} \omega_{i j} u_{j}\right)
$$

The main idea is to represent a multi-agent systems by a whole system. Matrix $\mathcal{A}=I_{n} \otimes A$ represents the system matrix and $\mathcal{B}=I_{n} \otimes B$ is the input matrix. If we denote $\hat{u}_{i}=u_{i}-\sum_{j \in \mathcal{N}_{i}} \omega_{i j} u_{j}$ The input vector is $\mathcal{U}^{T}=$ $\left[\hat{u}_{1}, \ldots, \hat{u}_{n}\right]$. We omit these uncertainties parts temporarily for the sake of studying the principle characteristics of the formation, then the nominal error dynamics for the MAS is given by

$$
\dot{\mathfrak{e}}=\mathcal{A} \mathfrak{e}+\mathcal{B U}
$$

The control input $\mathcal{U}$ is called the formation controller.

Substituting equation (15) into (17), the model of the whole system with rigid formation is given by

$$
\alpha(\mathcal{T}) \dot{\mathfrak{d}}=\mathcal{A} \alpha(\mathcal{T}) \mathfrak{d}+\mathcal{B U}
$$

In our work, the leaders and the followers do not have to keep a certain rigid communication structure in the group, they are organized based on the decentralized method, which depends on the neighbors of each agent. The formation shape and the communication structure of the group may be changing according to the position of each UAV. Therefore, the system in equation (18) will be a switched system as below

$$
\alpha\left(\mathcal{T}_{\sigma(t)}\right) \dot{\mathfrak{d}}=\mathcal{A} \alpha\left(\mathcal{T}_{\sigma(t)}\right) \mathfrak{d}+\mathcal{B U}
$$

Then (19) is the model of the multiple UAVs system with decentralized cooperation.

Remark 2. The model of the multiple UAVs with decentralized leader-follower cooperation is a switched system.

Remark 3. If the mapping matrix $\alpha\left(\mathcal{T}_{\sigma(t)}\right)$ is singular, the system will be a switched singular system.

Remark 4. If the mapping matrix is singular, the system (19) is not regular and unstable. 
In this subsection, the nominal model of the multi-UAVs formation has been proposed in equation (19), which is a switching system with the possibility of singular.

\section{DECENTRALIZED CONTROLLER DESIGN}

In this section, the decentralized controllers are designed and the consensus conditions are given. The scenarios is considered such that the topology is switching and the mapping matrix may be singular. The switching instants $\mathbb{T}=\left\{t_{1}, t_{2}, \ldots, t_{k}, \ldots\right\}$ can be rewritten as $\mathbb{T}=$ $\left\{\mathbb{T}_{1}, \ldots, \mathbb{T}_{l}, \ldots\right\}$, where $\mathbb{T}_{l}=\left\{t_{l 1}, \ldots, t_{l k_{l}}\right\}$ and $t_{l k_{l}}-t_{l 1}<$ $\infty$. Then, we are ready to introduce our main results in this work as the following theorem.

Theorem 1. For a leader-follower formation system, which has a nominal model in (19) with switching topology, the set of switching instants is given by $\mathbb{T}=$ $\left\{t_{11}, \ldots, t_{1 k_{1}}, \ldots, t_{l 1}, \ldots, t_{l k_{l}}, \ldots\right\}, k_{l} \geq 1$. All the agents achieve consensus, if

(i) The controller $\overline{\mathcal{U}}=\mathcal{U}+\mathcal{V}$ are used, where $\mathcal{U}$ has the same format as in equation (17) and $\mathcal{V}^{T}=$ $\left[v_{1}, \ldots, v_{n}\right]$. The decentralized controller $\hat{u}_{i}=\xi e_{i}$ is the control input for the error dynamics of agent $s_{i}$, $i \in \mathcal{V}$. For a leader, $e_{i}$ is

$e_{i}=\mu\left(s_{j}\right)\left[x_{i}-\mathcal{R}(t)\right]+\left[1-\mu\left(s_{j}\right)\right] x_{i}-\sum_{j \in \mathcal{N}_{i}} \omega_{i j} x_{j}(20)$

The feedback gain is $\xi=-\gamma B^{T} P$, where $\gamma$ is a scalar, $P=P^{T}>0$ and $Q=Q^{T}$, which satisfy the following Algebraic Riccati Equation (ARE)

$$
A^{T} P+P A-2 \gamma P B B^{T} P+Q=0
$$

(ii) The mapping matrix $\mathcal{T}(t)$ satisfies

$$
\operatorname{Rank}\left(\sum_{t_{j 1}}^{t_{j k_{j}}} \mathcal{T}_{\sigma(t)}\right)=n, \quad j=\{1, \ldots, l, \ldots\}
$$

where $n$ is the number of agents.

(iii) The states of agent $s_{i}$ can be stabilized in presence of the uncertainties by using the controller $\tilde{u}_{i}=u_{i}+v_{i}$, where $v_{i}$ is the Lyapunov redesign compensation for agent $s_{i}$, which is given by

$$
v_{i}=-\eta_{i}\left(\Theta_{i}, \dot{\Theta}_{i}, \mathcal{F}\right) \frac{w_{i}}{\left|w_{i}\right|}
$$

where $w_{i}=\frac{\partial V\left(e_{i}\right)}{\partial e_{i}} B, \eta_{i}$ is a nonnegative function w.r.t $\Theta_{i}, \dot{\Theta}_{i}$, and $\mathcal{F}$. The notation $\Theta_{i}$ represents the vector of Euler angles for agent $s_{i}$ and $\mathcal{F}$ is the variation of the thrust force.

Proof. The proof of the theorem is mainly divided into two parts. To begin with, the controller to stabilize the decoupled nominal model is proposed, using Lyapunov design. Furthermore, the uncertainties are considered by using Lyapunov redesign.

\section{a. Lyapunov design}

For each agent $s_{i}$, the error dynamics is given by equation (16). The Lyapunov function candidate for (16) is given by

$$
V\left(e_{i}\right)=e_{i}^{T} P e_{i}
$$

where $P=P^{T}>0$. The task is to find out an admissible controller $\hat{u}_{i}$, which satisfies

$$
\sup _{u_{i} \in R}\left\{\frac{\partial V\left(e_{i}\right)}{\partial e_{i}}\left(A e_{i}+B \hat{u}_{i}\right)\right\}<0, \forall e_{i} \neq 0
$$

Using equation (24), we can rewrite (25) as

$$
\begin{aligned}
\sup _{u_{i} \in R}\left\{e_{i}^{T} A^{T} P e_{i}+e_{i}^{T} P A e_{i}+\hat{u}_{i}^{T} B^{T} P e_{i}+e_{i}^{T} P B \hat{u}_{i}\right\} \\
<0, \forall e_{i} \neq 0
\end{aligned}
$$

According to condition (i),

$$
\hat{u}_{i}=\xi e_{i}=-\gamma B^{T} P e_{i}
$$

This is equivalent to minimize the cost function $J_{i}=$ $\int_{0}^{\infty} e_{i}^{T}(t) Q e_{i}(t)+\frac{1}{2 \gamma} \hat{u}_{i}^{2}(t) d t$

Substituting equation (27) into (26), thus, the problem becomes to find out a positive definite matrix $P$, which satisfies the following inequality with some $\gamma$.

$$
\sup _{\gamma \in R}\left\{A^{T} P+P A-2 \gamma P B B^{T} P\right\}<0
$$

Such a $P$ can be found by resolving the ARE in (21). Thus, for agent $s_{i}$, if we choose the Lyapunov function as $V\left(e_{i}\right)=e_{i}^{T} P e_{i}$, equation (21) guarantees that

$$
\dot{V}\left(e_{i}\right)=-e_{i}^{T} Q e_{i}
$$

where $Q$ is positive definite. Therefore, the closed-loop system is asymptotically stable.

According to definition $1, r(t)=\left(r_{X}(t), r_{Y}(t)\right)$ represents the external reference signal, which satisfies $r^{(4)}(t)=0$ and $P_{s_{i}}=(X, Y)$ represent the coordinates of agent $s_{i}$. A group of agents achieve consensus when conditions $\| X_{i}-$ $r_{X}(t), \ldots, X_{n}-r_{X}(t) \| \rightarrow 0$ and $\| Y_{i}-r_{Y}(t), \ldots, Y_{n}-$ $r_{Y}(t) \| \rightarrow 0$ are satisfied.

Let us denote $\mathcal{K}=I_{n} \otimes \xi_{i}$. According to equation (13), (14), and (20), we can rewrite the controller $\mathcal{U}$ for the model (19) as

$$
\mathcal{U}=\mathcal{K} \mathfrak{e}=\mathcal{K} \alpha\left(\mathcal{T}_{\sigma(t)}\right) \mathfrak{d}
$$

Then, the closed-loop system of (19) is rewritten by

$$
\alpha\left(\mathcal{T}_{\sigma(t)}\right) \dot{\mathfrak{d}}=(\mathcal{A}+\mathcal{B K}) \alpha\left(\mathcal{T}_{\sigma(t)}\right) \mathfrak{d}
$$

The mapping matrix changes at each switching instant. We call the model (19) between every two switches as a subsystem of the switching system. From the condition (ii), we obtain that on each time interval over $\mathbb{T}_{l}$, the summation of the corresponding sequence of these mapping matrices is nonsingular. We list these sub-systems during the time interval over $\mathbb{T}_{l}$ as follows

$$
\begin{gathered}
\alpha\left(\mathcal{T}_{\sigma\left(t_{l 1}\right)}\right) \dot{\mathfrak{d}}=(\mathcal{A}+\mathcal{B K}) \alpha\left(\mathcal{T}_{\sigma\left(t_{l 1}\right)}\right) \mathfrak{d} \\
\vdots \\
\alpha\left(\mathcal{T}_{\sigma\left(t_{l_{l} l}\right)}\right) \dot{\mathfrak{d}}=(\mathcal{A}+\mathcal{B K}) \alpha\left(\mathcal{T}_{\sigma\left(t_{l k_{l}}\right)}\right) \mathfrak{d}
\end{gathered}
$$

According to the property of Kronecker product, we have

$$
\sum_{j=1}^{k_{l}} \alpha\left(\mathcal{T}_{\sigma\left(t_{l j}\right)}\right)=\sum_{j=1}^{k_{l}} \mathcal{T}_{\sigma\left(t_{l j}\right)} \otimes I_{4}=\alpha\left(\sum_{j=1}^{k_{l}} \mathcal{T}_{\sigma\left(t_{l j}\right)}\right)
$$


Therefore, according to condition (ii),

$$
\operatorname{Rank}\left(\sum_{j=1}^{k_{l}} \alpha\left(\mathcal{T}_{\sigma\left(t_{l j}\right)}\right)\right)=4 \cdot \operatorname{Rank}\left(\sum_{j=1}^{k_{l}} \mathcal{T}_{\sigma\left(t_{l j}\right)}\right)=4 n
$$

In time interval $t_{l 1}<t<t_{(l+1) 1}$, we can choose a candidate Lyapunov function as

$$
V_{l}(\mathfrak{d})=\sum_{j=1}^{k_{l}} \mathfrak{d}^{T} \mathcal{P}_{l j} \mathfrak{d}
$$

where

$$
\mathcal{P}_{l j}=\left(\alpha\left(\mathcal{T}_{l j}\right) \otimes I_{m}\right)^{T} I_{n} \otimes P\left(\alpha\left(\mathcal{T}_{l j}\right) \otimes I_{m}\right)
$$

From the aforementioned analysis, we know that $V_{l}(\mathfrak{d})>0$, if $\mathfrak{d} \neq 0$. Thus, the Lyapunov function in (31) is feasible. Then, we have

$$
V_{l}(\mathfrak{d})=\sum_{j=1}^{k_{l}} \mathfrak{d}^{T} \mathcal{P}_{l j} \mathfrak{d}=\sum_{j=1}^{k_{l}} \mathfrak{e}^{T} I_{n} \otimes P \mathfrak{e}=\sum_{j=1}^{k_{l}} \sum_{i=1}^{n} V\left(e_{i}\right)
$$

The derivative of (31) is calculated as

$$
\dot{V}_{l}(\mathfrak{d})=\sum_{j=1}^{k_{l}} \sum_{i=1}^{n} \dot{V}\left(e_{i}\right)=\sum_{j=1}^{k_{l}} \sum_{i=1}^{n}-e_{i}^{T} Q e_{i} \leq 0
$$

This means that the tracking error $\mathfrak{d}$ converges to zero asymptotically in the time interval over $\mathbb{T}_{l}, l=\{1, \ldots\}$. For the switching time instant $t_{l k_{l}}$, since the equation (29) is always correct, then, we have

$$
V_{l}(\mathfrak{d})=\sum_{j=1}^{k_{l}} \sum_{i=1}^{n} V\left(e_{i}\left(t_{l k_{l}}\right)\right) \leq \sum_{j=1}^{k_{l}} \sum_{i=1}^{n} V\left(e_{i}\left(t_{l k_{l}}^{-}\right)\right)=V_{l-1}(\mathfrak{d})
$$

Then, by using the multiple Lyapunov function theorem for the switching system, the system is stable at these switching instants. Thus, the error $\mathfrak{d}$ will converge to the origin asymptotically without the effect of uncertainties.

\section{b. Lyapunov redesign}

For the purpose of overcoming the influence of unmodeled dynamics and the uncertainties, we propose to redesign the controller based on Lyapunov function.

By considering the uncertainties, the error dynamics can be rewritten as follows

$$
\dot{e}_{i}=A e_{i}+B\left(\hat{u}_{i}+v_{i}+\delta_{i}+\delta_{i}^{n b}\right)
$$

If we note $A^{\prime}=A-\gamma B^{T} P$, the error dynamics can be rewritten as

$$
\dot{e}_{i}=A^{\prime} e_{i}+B\left(v_{i}+\delta_{i}+\delta_{i}^{n b}\right)
$$

We may consider that $\left\|\delta_{i}^{n b}\right\|$ is bounded. Let us denote these uncertainties by the following expression

$$
\Delta_{i}=\delta_{i}+\delta_{i}^{n b}
$$

According to equations (4), (6), and (7), we can see that the uncertainties have the properties as the following inequality

$$
\begin{aligned}
\left|\Delta_{i}\right| & =\left|\delta_{i}\left(\Theta_{i}, \dot{\Theta}_{i}, \mathcal{F}, v_{i}\right)+\delta_{i}^{n b}\right| \\
& \leq \rho\left(\Theta_{i}, \dot{\Theta}_{i}, \mathcal{F}\right)+k_{i}\left|\ddot{\Theta}_{i}\right|+M_{i}^{n b} \\
& =\rho\left(\Theta_{i}, \dot{\Theta}_{i}, \mathcal{F}\right)+k_{i}\left|v_{i}\right|+M_{i}^{n b}
\end{aligned}
$$

where $\Theta_{i}=\left[\phi_{i}, \theta_{i}, \psi_{i}\right]^{T}$ represents the vector of Euler angles for agent $s_{i}$ and $\mathcal{F}^{T}=\left[\Delta_{F_{T}}, \dot{\Delta}_{F_{T}}, \ddot{\Delta}_{F_{T}}\right]$ is the variation of the thrust force. $\rho: \mathbb{R}^{9} \rightarrow \mathbb{R}$ is a nonnegative continuous function. The positive scalar $M_{i}^{n b}$ is the bound of the uncertainties of $\delta_{i}^{n b}$.

According to condition (i) and (ii), the controller $\mathcal{U}$ can stabilize the nominal system. Since in the first part of the controller design without considering the uncertainties, the stability of the nominal switching system is proven.

The nominal model is piecewise continuous in $t$, and Lipschitz in $x$ and $u$. For agent $s_{i}$, the Lyapunov function in equation (24) satisfies

$$
\begin{aligned}
& \dot{V}\left(e_{i}\right)=\frac{\partial V\left(e_{i}\right)}{\partial e_{i}}\left[A e_{i}+B\left(u_{i}+v_{i}+\Delta_{i}\right)\right] \\
& =\frac{\partial V\left(e_{i}\right)}{\partial e_{i}}\left[A e_{i}+B u_{i}\right]+\frac{\partial V\left(e_{i}\right)}{\partial e_{i}} B\left(v_{i}+\Delta_{i}\right) \\
& =-e_{i}^{T} Q e_{i}+\frac{\partial V\left(e_{i}\right)}{\partial e_{i}} B\left(v_{i}+\Delta_{i}\right) \\
& \leq \frac{\partial V\left(e_{i}\right)}{\partial e_{i}} B\left(v_{i}+\Delta_{i}\right)
\end{aligned}
$$

Let us denote $w_{i}=\frac{\partial V\left(e_{i}\right)}{\partial e_{i}} B$, and rewrite the last inequality as

$$
\begin{aligned}
\dot{V}\left(e_{i}\right) & \leq w_{i} v_{i}+w_{i} \Delta_{i} \\
& \leq w_{i} v_{i}+\left|w_{i}\right|\left[\rho\left(\Theta_{i}, \dot{\Theta}_{i}, \mathcal{F}\right)+M_{i}^{n b}+k_{i}\left|v_{i}\right|\right]
\end{aligned}
$$

In the following part, we make $\rho_{i}$ as an abbreviation of $\rho\left(\Theta_{i}, \dot{\Theta}_{i}\right)$. Let us take

$$
v_{i}=-\eta_{i}\left(\Theta_{i}, \dot{\Theta}_{i}, \mathcal{F}\right) \operatorname{sign}\left(w_{i}\right)
$$

Then, the derivative of the Lyapunov function satisfies

$$
\begin{aligned}
\dot{V}\left(e_{i}\right) & \leq-\eta_{i}\left|w_{i}\right|+k_{i}\left|w_{i}\right| \eta_{i}+\left|w_{i}\right|\left(\rho_{i}+M_{i}^{n b}\right) \\
& =-\left(1-k_{i}\right) \eta_{i}\left|w_{i}\right|+\left|w_{i}\right|\left(\rho_{i}+M_{i}^{n b}\right)
\end{aligned}
$$

If we choose $\eta_{i}$ as follows

$$
\eta_{i} \geq\left(\rho_{i}+M_{i}^{n b}\right) /\left(1-k_{i}\right)
$$

Then, the Lyapunov function yields

$$
\dot{V}\left(e_{i}\right) \leq-\left(1-k_{i}\right) \eta_{i}\left|w_{i}\right|+\left|w_{i}\right|\left(1-k_{i}\right) \eta_{i}=0
$$

The Lyapunov function for the overall system in equation (31) satisfies

$$
\dot{V}_{l}(\mathfrak{d})=\sum_{j=1}^{k_{l}} \sum_{i=1}^{n} \dot{V}\left(e_{i}\right) \leq 0
$$

Based on the analysis above, if we choose the controller for agent $s_{i}$ as $\mathcal{U}=\overline{\mathcal{U}}+\mathcal{V}$, where $\mathcal{U}=\left[\hat{u}_{1}, \hat{u}_{2}, \ldots, \hat{u}_{n}\right]^{T}$ and $\mathcal{V}=\left[v_{1}, v_{2}, \ldots, v_{n}\right]^{T}$.

The overall system will be asymptotically stable even in presence of uncertainties. It means that all the agents achieve consensus. This ends the proof.

Remark 5. The notation $\overline{\mathcal{U}}$ gathers the controllers for all the agents. It is for the convenience of stability proof, rather than a centralized controller. The centralized controller does not exist in our formation strategy.

Remark 6. If one agent can obtain the information of the reference command $r(t)$, it has the ability to behave as 
a leader or follower and it depends on the factor $\varepsilon_{i}$. For example, the value of $\varepsilon_{i}$ can depend on the recognition rate of the reference. When the sensor of camera is used to capture the target object, $\varepsilon_{i}$ represents the probability of recognition. The assignment of $\varepsilon_{i}$ is one part of our future work.

\section{SIMULATION}

In this section, we have mainly two tasks. To begin with, we will illustrate the problem that we have stated in introduction. Furthermore, the simulation results show the performance of the controller. In the following simulations, the formation system has seven agents and agent $s_{1}$ acts as a leader and agents $s_{2}, \ldots, s_{7}$ are followers. The reference command is represented by $r(t)=\left(k_{X} t, k_{Y} t\right)$, where $k_{X}=$ $k_{Y}=1$.

Taking $\gamma=1$ and the symmetric positive definite matrix $P$ as

$$
P=\left[\begin{array}{cccc}
26.8247 & 30.9783 & 17.2024 & 3.1623 \\
30.9783 & 65.8961 & 42.9828 & 8.4827 \\
17.2024 & 42.9828 & 44.8076 & 9.7962 \\
3.1623 & 8.4827 & 9.7962 & 5.4399
\end{array}\right]
$$

One solves (21) for $Q$ giving

$$
Q=\left[\begin{array}{llll}
20.0000 & 26.8247 & 30.9783 & 17.2024 \\
26.8247 & 81.9566 & 83.0985 & 46.1451 \\
30.9783 & 83.0985 & 105.9657 & 53.2903 \\
17.2024 & 46.1451 & 53.2903 & 39.5924
\end{array}\right]
$$

which can be verified to be a symmetric positive definite matrix. This gives rise to the following control gain values

$$
\xi=\left[\begin{array}{llll}
-3.1623-8.4827 & -9.7962 & -5.4399
\end{array}\right]
$$

which we used in our simulations.

In Fig. 1, the formation can not achieve consensus, if we use the ordinary decentralized controller without considering the influence of uncertainties. Conversely, the formation achieves consensus by using the controller based on Lyapunov design and redesign, which has been shown in Fig. 2.

Both in Fig. 1 and Fig. 2, the mapping matrices are fixed and nonsingular.

In Fig. 3 and Fig. 4, the initial conditions for each agents are $x_{1}=[0,0,0,0]^{T}, x_{2}=[21,0,0,0]^{T}, x_{3}=[16,0,0,0]^{T}$, $x_{4}=[7,0,0,0]^{T}, x_{5}=[2,0,0,0]^{T}, x_{6}=[-10,0,0,0]^{T}$, $x_{7}=[-11,0,0,0]^{T}$. In Fig. 3, the mapping matrix $\mathcal{T}_{b}$ is not full rank. The condition (ii) in theorem 1 is not satisfied. In Fig. 4, the mapping matrix switches from $\mathcal{T}_{b}$ to $\mathcal{T}_{a} \cdot \mathcal{T}_{a}$ and $\mathcal{T}_{b}$ are written as follows

$\mathcal{T}_{a}=\left[\begin{array}{ccccccc}1 & 0 & 0 & 0 & 0 & 0 & 0 \\ 0 & 1 & -1 & 0 & 0 & 0 & 0 \\ 0 & 0 & 1 & -1 & 0 & 0 & 0 \\ 0 & 0 & 0 & 1 & -1 & 0 & 0 \\ -1 & 0 & 0 & 0 & 1 & 0 & 0 \\ -1 & 0 & 0 & 0 & 0 & 1 & 0 \\ -1 & 0 & 0 & 0 & 0 & 0 & 1\end{array}\right] \quad \mathcal{T}_{b}=\left[\begin{array}{ccccccc}1 & 0 & 0 & 0 & 0 & 0 & 0 \\ 0 & 1 & -1 & 0 & 0 & 0 & 0 \\ 0 & 0 & 1 & -1 & 0 & 0 & 0 \\ 0 & -1 & 0 & 1 & 0 & 0 & 0 \\ -1 & 0 & 0 & 0 & 1 & 0 & 0 \\ -1 & 0 & 0 & 0 & 0 & 1 & 0 \\ -1 & 0 & 0 & 0 & 0 & 0 & 1\end{array}\right]$

We can see that in Fig. 3, the group of agents can not achieve consensus, because the condition (ii) in theorem 1 is not satisfied. On the contrary, in Fig. 4 the topology switched from $\mathcal{T}_{b}$ to $\mathcal{T}_{a}$, then the conditions in theorem 1 are satisfied, thus, all the agents keep consensus.

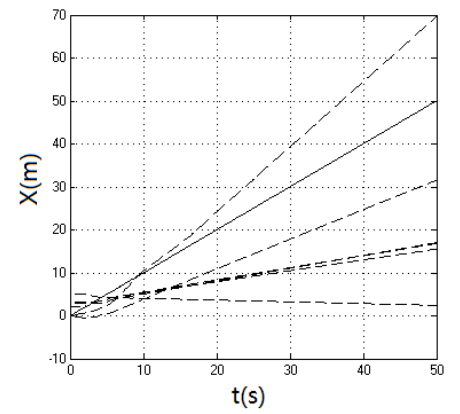

Fig. 1. The tracking curve with uncertainties by using simplified model based controller design

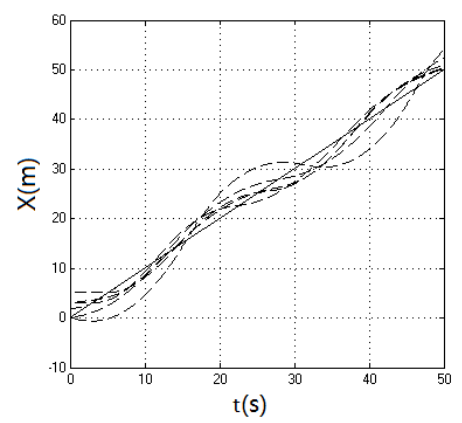

Fig. 2. The tracking curve with uncertainties by using Lyapunov design and redesign

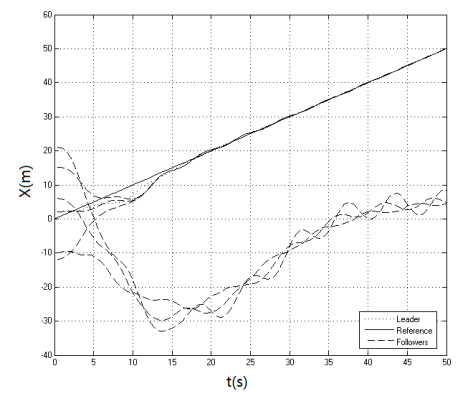

Fig. 3. The tracking curve when the topology is fixed and the mapping matrix is singular

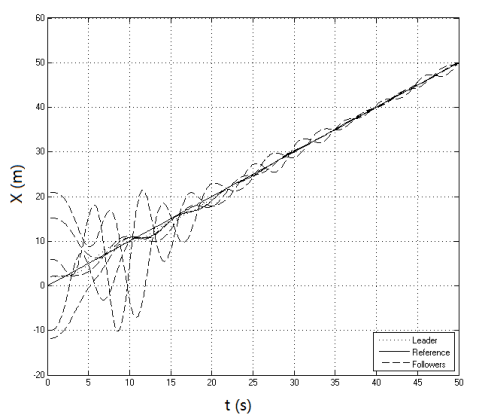

Fig. 4. The tracking curve with switching topology 


\section{CONCLUSION}

In this paper, we propose a decentralized control strategy for the leader-follower formation with nonlinear and coupled agent dynamics. The main idea is to represent the agent dynamics by a nominal decoupled linear model, and then, analyze the probable uncertainties. We propose a switched singular system to model the formation with switching topology. By using Lyapunov design and redesign method, we prove the consensus of the agents even in presence of switching topology and model uncertainties.

\section{REFERENCES}

Bresciani, T. (2008). Modelling, Identification and Control of a Quadrotor Helicopter. Lund University.

Cao, Y., Ren, W., and Egerstedt, M. (2012). Distributed containment control with multiple stationary or dynamic leaders in fixed and switching directed networks. Automatica, 48(8), 1586-1597.

Guo, W., Lu, J., Chen, S., and Yu, X. (2011). Second-order tracking control for leader-follower multi-agent flocking in directed graphs with switching topology. Systems and Control Letters, 60(12), 1051 - 1058.

Hong, Y., Hu, J., and Gao, L. (2006). Tracking control for multi-agent consensus with an active leader and variable topology. Automatica, 42(7), 1177-1182.

Li, Z., Ren, W., Liu, X., and Fu, M. (2013). Distributed containment control of multi-agent systems with general linear dynamics in the presence of multiple leaders. International Journal of Robust and Nonlinear Control, $23,534-547$.

Memon, A. and Khalil, H. (2008). Lyapunov redesign approach to output regulation of nonlinear systems using conditional servocompensators. In American Control Conference, 2008, 395-400.

Ni, W. and Cheng, D. (2010). Leader-following consensus of multi-agent systems under fixed and switching topologies. Systems and Control Letters, 59(3-4), 209-217.

Notarstefano, G., Egerstedt, M., and Haque, M.A. (2011). Containment in leader-follower networks with switching communication topologies. Automatica, 47(5), 10351040 .

Porfiri, M., Roberson, D.G., and Stilwell, D.J. (2007). Tracking and formation control of multiple autonomous agents: A two-level consensus approach. Automatica, 43(8), $1318-1328$.

Qu, Z., Wang, J., and Hull, R. (2008). Cooperative control of dynamical systems with application to autonomous vehicles. IEEE Transactions on Automatic Control, 53(4), 894-911.

Vela, P., Betser, A., Malcolm, J., and Tannenbaum, A. (2008). Vision-based range regulation of a leaderfollower formation. IEEE Transactions on Control Systems Technology, 17(2), 442-448.

Wang, J. and $\mathrm{Hu}$, X. (2010). Observer-based tracking problem of leader-follower multi-agent systems. In 29th Chinese Control Conference (CCC), 2010, 5821-5826.

You, K., Li, Z., and Xie, L. (2013). Consensus condition for linear multi-agent systems over randomly switching topologies. Automatica, 49(10), 3125 - 3132 . 